Revue des sciences de l'eau

\title{
Évaluation de la capacité de rétention des métaux dissous d'un marais artificiel en utilisant le périphyton et le gastéropode Helisoma trivolvis \\ The use of periphyton and the freshwater gastropod Helisoma trivolvis to assess dissolved metal retention by a constructed wetland
}

\author{
M. Simonyi-Poirier, R. R. Goulet et F. R. Pick
}

Volume 16, numéro 2, 2003

URI : https://id.erudit.org/iderudit/705506ar

DOI : https://doi.org/10.7202/705506ar

Aller au sommaire du numéro

Éditeur(s)

Université du Québec - INRS-Eau, Terre et Environnement (INRS-ETE)

ISSN

0992-7158 (imprimé)

1718-8598 (numérique)

Découvrir la revue

Citer cet article

Simonyi-Poirier, M., Goulet, R. R. \& Pick, F. R. (2003). Évaluation de la capacité de rétention des métaux dissous d'un marais artificiel en utilisant le périphyton et le gastéropode Helisoma trivolvis. Revue des sciences de l'eau/ Journal of Water Science, 16(2), 237-254. https://doi.org/10.7202/705506ar

\section{Résumé de l'article}

Le but de cette étude est de déterminer si le périphyton et le gastéropode pulmoné Helisoma trivolvis peuvent être utilisés pour évaluer la capacité de rétention des métaux dissous d'un marais construit. Cette étude a été menée dans un marais de la région d'Ottawa-Carleton (Ontario, Canada), qui a été construit en 1995 afin d'améliorer la qualité des eaux de ruissellement provenant d'un bassin versant à usage résidentiel et agricole. Au cours du mois de septembre 1999, des échantillons d'eau ont été prélevés, des escargots ( $H$. trivolvis) ont été recoltés et un substrat artificiel a été utilisé pour faire croître du périphyton, à l'entrée et à la sortie d'eau du marais. En moyenne, les calculs de balance de masse indiquaient une rétention des formes dissoutes du $\mathrm{Cu}, \mathrm{Mn}$, $\mathrm{Ni}$ et $\mathrm{Zn}$ dans le marais. Cependant, les tendances observées pour les concentrations de métaux dissous et pour les concentrations dans les organismes différaient pour certains métaux. Les concentrations de $\mathrm{Cd}$ et $\mathrm{Ni}$ dans les tissus de $H$. trivolvis et le périphyton étaient significativement plus élevées à l'entrée qu'à la sortie. Cependant, les concentrations de $\mathrm{Cr}$ et $\mathrm{Al}$ dans les organismes n'étaient pas significativement différentes entre l'entrée et la sortie alors que celle de Mn était significativement plus élevée à la sortie du marais. Pour tous les métaux sauf le $\mathrm{Cd}$ et le $\mathrm{Zn}$, les concentrations dans le périphyton étaient en moyenne plus elevées que celles dans les escargots. Le périphyton peut donc fournir une mesure plus conservatrice de la contamination du milieu pas les métaux. Cette étude montre l'importance de considérer, non-seulement les mesures chimiques, mais aussi les mesures biologiques, dans l'évaluation de l'efficacité d'un ouvrage de contrôle de pollution. 


\title{
Évaluation de la capacité de rétention des métaux dissous d'un marais artificiel en utilisant le périphyton et le gastéropode Helisoma trivolvis
}

\author{
The use of periphyton and the freshwater gastropod \\ Helisoma trivolvis to assess dissolved metal retention \\ by a constructed wetland
}

\section{SIMONYI-POIRIER, R. R. GOULET et F. R. PICK*}

Reçu le 4 juillet 2000, accepté le 29 janvier 2003**.

\section{SUMMARY}

\begin{abstract}
Urban and agricultural development has had a significant impact on the water quality of rivers and lakes around the world. In the last few decades, constructed wetlands have been designed as wastewater treatment systems to prevent water quality deterioration in natural receiving waters. Constructed wetlands are built because they are considered sinks for many pollutants thereby protecting the water quality of downstream ecosystems. The treatment performance of these wetlands is generally assessed using mass balance calculations. However, the retention of metals by constructed wetlands is highly variable and the factors involved are still poorly understood. If wetlands are sinks for metals, the metal content of organisms should be lower downstream than upstream. In this context, organisms can be useful to assess the retention or transformation of metals by wetlands.
\end{abstract}

The objective of this study was to determine whether periphyton and the gastropod Helisoma trivolvis Say could be used to evaluate the retention of dissolved metals in a constructed wetland. $H$. trivolvis is a freshwater pulmonate snail widespread in ponds across North America. It feeds mostly on periphyton and is more or less sedentary. Snails have been used as biomonitors because several species are metal tolerant. However, compared to snails, periphytic microorganisms may track more closely dissolved metal concentrations as they take up metals principally from the water column.

This study was conducted at the Monahan Pond in Kanata, Ontario (Canada). This wetland was built in 1995 to treat run-off from an agricultural and residential watershed. Water chemistry samples, snails and periphyton grown on artificial substrata were collected at both the inlet and the

Institut de Biologie d'Ottawa-Carleton, Université d'Ottawa, 30 Marie-Curie, Ottawa, Ontario, Canada, K1N 6N5. Té. I: (613) 562-5800 poste 6365. Télécopieur: (613) 562-5486,

* Correspondance. E-mail : frpick@science.uottawa.ca

** Les commentaires seront reçus jusqu'au 30 décembre 2003. 
outlet of the wetland in the fall of 1999 . Tissue samples were digested with concentrated nitric acid and metal analyses were done by ICP-MS (Inductively Coupled Plasma Mass Spectrometry). During the experiment several chemical parameters differed between the inlet and the outlet. Alkalinity was significantly higher at the inlet and all major cation concentrations were higher at the inlet. The temperature was on average $3{ }^{\circ} \mathrm{C}$ higher at the outlet. Mass balance calculations showed that the wetland was a sink for most dissolved metals. Snails and periphyton tissue metal concentrations were higher at the inlet than at the outlet for Cd and Ni. However, no significant differences were observed between inlet and outlet tissue concentrations for $\mathrm{Cr}$ and $\mathrm{Al}$, whereas Mn was actually significantly higher at the outlet. As a result, the metal content of the organisms did not consistently reflect the dissolved metal concentrations in water. For all metals except $\mathrm{Cd}$ and $\mathrm{Zn}$, periphyton concentrations were on average higher than snail metal concentrations. Periphyton analyses can provide a more conservative measure of metal contamination and, when artificial substrates are used, correspond to defined and known periods of exposure.

This study demonstrates that constructed wetlands may lead to increased metal content of downstream organisms even if these wetlands appear to be overall sinks for dissolved metals based on mass balance calculations. It also shows the need to consider not only metal concentrations, but also biological data when assessing the performance of pollution control facilities.

Key words: constructed wetlands, treatment efficiency, metals, periphyton, pulmonate snail, Helisoma trivolvis.

\section{RÉSUMÉ}

Le but de cette étude est de déterminer si le périphyton et le gastéropode pulmoné Helisoma trivolvis Say peuvent être utilisés pour évaluer la capacité de rétention des métaux dissous d'un marais construit. Cette étude a été menée dans un marais de la région d'Ottawa-Carleton (Ontario, Canada), qui a été construit en 1995 afin d'améliorer la qualité des eaux de ruissellement provenant d'un bassin versant à usage résidentiel et agricole. Au cours du mois de septembre 1999, des échantillons d'eau ont été prélevés, des escargots $(H$. trivolvis) ont été recoltés et un substrat artificiel a été utilisé pour faire croître du périphyton, à l'entrée et à la sortie d'eau du marais. En moyenne, les calculs de balance de masse indiquaient une rétention des formes dissoutes du $\mathrm{Cu}, \mathrm{Mn}, \mathrm{Ni}$ et $\mathrm{Zn}$ dans le marais. Cependant, les tendances observées pour les concentrations de métaux dissous et pour les concentrations dans les organismes différaient pour certains métaux. Les concentrations de $\mathrm{Cd}$ et $\mathrm{Ni}$ dans les tissus de $\boldsymbol{H}$. trivolvis et le périphyton étaient significativement plus élevées à l'entrée qu'à la sortie. Cependant, les concentrations de $\mathrm{Cr}$ et $\mathrm{Al}$ dans les organismes n'étaient pas significativement différentes entre l'entrée et la sortie alors que celle de Mn était significativement plus élevée à la sortie du marais. Pour tous les métaux sauf le $\mathrm{Cd}$ et le $\mathrm{Zn}$, les concentrations dans le périphyton étaient en moyenne plus elevées que celles dans les escargots. Le périphyton peut donc fournir une mesure plus conservatrice de la contamination du milieu pas les métaux. Cette étude montre l'importance de considérer, non-seulement les mesures chimiques, mais aussi les mesures biologiques, dans l'évaluation de l'efficacité d'un ouvrage de contrôle de pollution.

Mots clés : efficacité de traitement, gastéropode pulmoné, Helisoma trivolvis, marais artificiels, métaux, périphyton. 


\section{1 - INTRODUCTION}

Les marais construits sont des systèmes utilisés pour le traitement des eaux usées. Ils représentent une alternative peu coûteuse et facile à opérer par rapport aux systèmes conventionnels de traitement des eaux usées (KADLEC et KNIGHT, 1996). Ils peuvent modifier, transformer et retenir de nombreux contaminants par une combinaison de processus biologiques, chimiques et hydrologiques (KADLEC et KNIGHT, 1996; MITSCH et GOSSELINK, 2000; SCHOLES et al., 1998). La quantité de contaminants qu'ils reçoivent varie selon l'utilisation du bassin versant, la superficie de drainage, la fréquence des précipitations et plusieurs autres facteurs. Les principaux polluants retrouvés dans les eaux de ruissellement d'origine urbaine sont les solides en suspension, les métaux lourds et les coliformes fécaux (BREEN et al., 1994; HELFIELD et DIAMOND, 1997). Les marais construits peuvent aussi servir au traitement de polluants d'origine agricole, comme les excès de phosphore et d'azote (MITSCH et GosSELINK, 2000; REDDY et al., 1999 ; WOOD et MCATAMNEY, 1996). Ils sont utilisés pour le traitement des métaux contenus dans les eaux usées et dans le drainage minier acide (HAWKINS et al., 1997). Cependant, la rétention des métaux par les marais construits est variable et les facteurs expliquant cette variabilité sont encore peu connus (KADLEC et KNIGHT, 1996).

De façon générale, la rétention des métaux par ces marais est évaluée en comparant la masse de métaux à l'entrée et à la sortie (KADLEC et KNIGHT, 1996 ; MITSCH et GOSSELINK, 2000; SCHOLES et al., 1998). Cette méthode pose deux problèmes: premièrement la prise d'échantillons n'est souvent pas assez fréquente car les concentrations en métaux des eaux de surface peuvent varier de façon importante à l'intérieur d'une période de 24 heures (GOULET et PICK, 2001; WIEDER, 1994). II est donc nécessaire d'augmenter le nombre d'échantillons d'eau afin de mieux évaluer la rétention des métaux par les marais. Deuxièmement, les budgets de masse sont généralement basés sur des quantités de métaux totaux (sous forme dissoute et particulaire) dans l'eau et ces budgets ne permettent pas d'évaluer la biodisponibilité des métaux aux organismes vivants.

Le traitement des eaux usées par marais a pour but premier de minimiser l'exposition des organismes aux métaux qui peuvent s'avérer toxiques lorsque leurs concentrations atteignent des niveaux élevés. Curieusement, dans une étude antérieure, nous avons observé que les concentrations de métaux dans le gastéropode Helisoma trivolvis, étaient souvent plus élevées à la sortie qu'à l'entrée du marais et ce, malgre le fait que le marais retenait les métaux dissous (GoULET et al., 2001a). Nous avons expliqué ce résultat par le fait que le marais était une source de métaux sous forme particulaire, potentiellement biodisponible aux gastéropodes qui peuvent puiser les métaux, non seulement de l'eau de surface, mais aussi de leur nourriture (FLESSAS et al., 2000). Or, il n'est pas évident que les gastéropodes étaient effectivement exposés à cette forme particulaire provenant de l'eau de surface. II est possible que les teneurs en métaux plus elevées observées dans les gastéropodes (GOULET et al., 2001a) soient le résultat d'une nourriture plus contaminée à la sortie du marais. $H$. trivolvis est un gastéropode brouteur détritivore et algivore, qui se nourrit principalement du périphyton et des détritus (BROWN, 1991; SMITH, 1989). Hors, il est bien connu que les algues du périphyton ont la capacité d'accumuler les métaux à des concentrations élevées (ENGDAHI et al., 1998; SANDERS et RIEDEL, 1998; VYMAZAL, 1995). 
L'objectif de cette étude était de déterminer si les concentrations en métaux dissous pouvaient être reliées aux teneurs en métaux du périphyton, et si ces dernières pouvaient être reliées au contenu en métaux des gastéropodes situés en amont et en aval d'un marais construit. Si le marais retenait les métaux biodisponibles les concentrations de métaux dans le périphyton et dans les escargots devraient être plus élevées à l'entrée qu'à la sortie d'eau du marais.

\section{2 - MATÉRIEL ET MÉTHODE}

\subsection{Site de l'étude}

Cette étude a été menée au marais Monahan situé au sud de la ville de Kanata (45016' $N, 75^{\circ} 51^{\prime} \mathrm{W}$ ) (fig. 1). Le marais a été construit en 1995 dans le but d'améliorer la qualité des eaux de ruissellement provenant du bassin versant à usage résidentiel et agricole. La surface de drainage du marais est d'environ 637 hectares $\left(637 \times 10^{4} \mathrm{~m}^{2}\right)$, son volume maximal, de $5,4 \times 10^{4} \mathrm{~m}^{3}$ et sa profondeur moyenne, de $1,5 \mathrm{~m}$. Le temps de résidence moyen de l'eau dans le marais est de $12,7 \pm 0,8$ jours. Le marais est constitué de trois cellules qui ont été initialement recouvertes d'une couche d'argile pour prévenir l'infiltration (figure 1) (CITY OF KANATA, 1997). Des quenouilles (Typha latifolia) ont été plantées sur le substrat d'argile et le marais a été inondé en 1996 pour sa première année d'opération. Depuis, les quenouilles sont abondantes dans le littoral et des plantes aquatiques (Lemna minor) ont colonisé le marais. Le bassin versant est surtout agricole, mais le secteur résidentiel est en développement rapide (CITY OF KANATA, 1998).

\subsection{Collecte des échantillons}

La collecte des données sur le terrain a été effectuée au cours du mois de septembre 1999.

\subsubsection{Périphyton}

Pour minimiser la variation causée par le substrat naturel, un substrat artificiel en argile $(9,7 \times 9,7 \mathrm{~cm})$ a été utilisé pour faire croître le périphyton. 40 tuiles au total ont été déposées dans le fond du marais: 20 à l'entrée et 20 à la sortie du marais. La période de croissance du périphyton a été de 20 jours. Lors de la récolte des tuiles (le 23 septembre 1999), 17 tuiles sur 20 ont été recupérées à l'entrée et 16 sur 20 à la sortie du marais. Les tuiles ont été rincées plusieurs fois avec l'eau du site pour enlever les dépôts de sédiments sur les tuiles et celles-ci ont été disposées séparément dans des sacs de plastique Ziploc pour être ensuite ramenées au laboratoire. Au laboratoire, le périphyton a été rincé plusieurs fois avec l'eau MilliQ et le périphyton a été enlevé de la surface de chaque tuile à l'aide d'une spatule en Téflon préalablement lavée à l'acide. Le périphyton récolté sur chaque tuile a été congelé dans une solution d'eau MilliQ dans un contenant individuel de polyéthylène de haute densité. 
A)
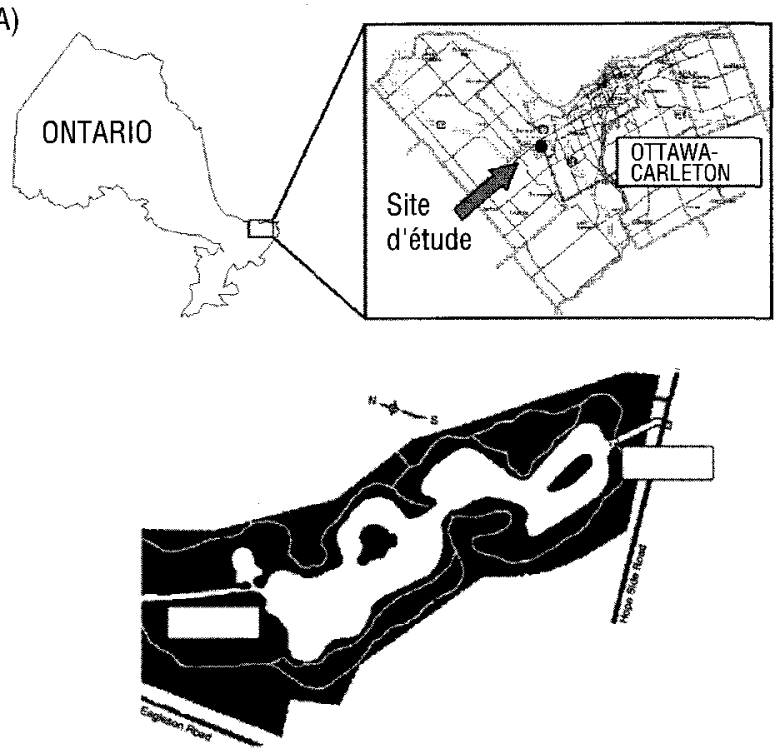

Figure 1 A) Emplacement géographique du marais Monahan, Kanata, Ontario et $B)$ représentation schématique du marais Monahan. Les sites de récolte des spécimens et de l'eau ont été fait à l'entrée et à la sortie du marais.

A) Geographical location of the Monahan Pond, Kanata, Ontario and B) map of the Monahan wetland. Periphyton, gastropods and water samples were collected at the inlet and outlet of the wetland.

\subsubsection{Helisoma trivolvis}

Lors de la récolte du périphyton, des gastéropodes ont été récoltés de façon aléatoire à l'entrée et à la sortie du marais. Dix-huit gastéropodes à l'entrée et 13 à la sortie du marais ont été récoltés, le 13 septembre 1999. Les spécimens ont été récoltés sur les substrats rocheux ou les structures de béton, puis rincés et placés dans des sacs en plastique scellés Ziploc. On les a ensuite apportés au laboratoire et transférés dans des contenants en polyéthylène de haute-densité où ils ont été immédiatement congelés. Le diamètre de la coquille de chacun des gastéropodes a été mesuré pour déterminer si les populations à l'entrée et à la sortie étaient similaires en terme de taille, étant donné que la teneur en métaux des organismes peut en être affectée (BOYDEN, 1974 ; COUGHTREY et MARTIN, 1977).

\subsubsection{Eau}

Pendant la période de croissance, les précipitations ont été fréquentes. Les données météorologiques ont été obtenues de la station météorologique d'Environnement Canada de la région d'Ottawa-Carleton. Le temps de résidence de l'eau a été calculé durant cette période (tableau 1) selon la formule suivante:

$$
t=V / Q_{t}
$$


où $t$ représente le temps de résidence (j), $Q_{t}$ représente le débit à l'entrée du marais $\left(\mathrm{m}^{3} \mathrm{j}^{-1}\right)$ et $\mathrm{V}$ représente le volume moyen d'eau dans le marais $\left(\mathrm{m}^{3}\right)$ (MITSCH et GOSSELINK, 2000).

Durant la période de croissance du périphyton, plusieurs paramètres chimiques de l'eau ont été mesurés et des échantillons d'eau ont été pris à tous les trois jours. Nous avons prélévé des échantillons d'eau pour déterminer les concentrations en métaux dissous et de certains cations dans le marais (2 réplicats à l'entrée et 2 réplicats à la sortie lors de chaque journée d'échantillonnage). Les échantillons d'eau ont été recueillis dans des bouteilles Nalgene de polyéthylène de haute densité (HDPE) de $60 \mathrm{ml}$. Les échantillons d'eau ont été filtrés sur le terrain avec des filtres de polypropylène de Nylon de $0,45 \mu \mathrm{m}$ (Chromatographic Specialties Inc.), en utilisant une seringue de $50 \mathrm{~mL}$ (Markson Ltd.) sans ventouse de caoutchouc, pour éviter toute contamination. Les échantillons d'eau ont été entreposés à $4^{\circ} \mathrm{C}$ au laboratoire jusqu'à l'analyse par ICP-MS (Inductively Coupled Plasma - Mass Spectrometry) (Commission Géologique du Canada). À l'aide d'une sonde multi-paramètres (Hydrolab MiniSonde et Surveyor 4), nous avons mesuré le $\mathrm{pH}$, la température, la concentration d'oxygène dissous, et la conductivité de l'eau à l'entrée et à la sortie, lors de chaque sortie sur le terrain. L'alcalinité a été déterminée à l'aide de la méthode de titration de Gran (WETZEL et LIKENS, 1991).

Des échantillons d'eau ont aussi été prélévés dans des contenants de polyéthylène de haute densité pour déterminer la concentration totale de carbone organique dissous (COD) et la concentration de certains anions majeurs dans l'eau (2 réplicats à l'entrée et 2 réplicats à la sortie lors de chaque période d'échantillonnage). Les échantillons d'eau servant à mesurer le COD ont d'abord été filtrés (filtres de pores de $0,45 \mu \mathrm{m}$ de type $\mathrm{HA}$ ) et ont ensuite été analysés avec un Hewlett Packard DC-190 TOC Analyser, en utilisant deux standards de glucose (10 et $20 \mathrm{mg} / \mathrm{L}$ ) pour calibrer l'appareil. Les échantillons d'eau destinés à l'analyse des anions ont été conservés à $4^{\circ} \mathrm{C}$ jusqu'à l'analyse par chromatographie d'ions avec un DIONEX DX-100 Ion Chromatograph.

\subsection{Analyses en laboratoire}

\subsubsection{Digestions}

Les gastéropodes ont d'abord été séparés de leur coquille. Le périphyton et les gastéropodes ont ensuite été déshydratés par lyophilisation (Labconco Freeze Dryer). Chaque gastéropode a été pesé (masse sèche) et ensuite digéré par micro-onde (CEM model MDS-2000) (CEM bovine liver microwave digestion method) dans $10 \mathrm{~mL}$ d'acide nitrique concentré de haute pureté (Seastar Chemicals Inc., Vancouver), dans des tubes de Téflon. La durée de la digestion était de 30 minutes au total pour atteindre une pression maximale de 1030 kPA. Dans certains cas, nous avons groupé deux escargots pour obtenir une masse sèche minimum de $0,1 \mathrm{~g}$ par échantillon pour les digestions. Seize échantillons d'escargots ont été analysés au total. Les escargots n'ont pas été purgés (élimination des métaux contenus dans leur système digestif), mais il a été démontré que la différence entre les organismes purgés et non purgés n'étaient pas toujours significative pour d'autres espèces d'escargots (FLESSAS et al., 2000). 
Pour la digestion du périphyton, un maximum de $0,5 \mathrm{~g}$ de masse sèche de chaque échantillon a été utilisé. Chaque échantillon a ensuite été digéré séparément dans le four aux micro-ondes (CEM model MDS-2000) (CEM alfalfa microwave digestion method) avec de l'acide nitrique concentré de haute pureté, dans des tubes de Téflon. La durée de la digestion était de 22 minutes pour atteindre une pression maximale de $1030 \mathrm{kPa}$. Au total, 32 échantillons de périphyton ont été digérés. Après une période de refroidissement de 30 minutes, les contenants de Téflon ont été ouverts et les solutions diluées ( $1 \mathrm{ml}$ de solution dans $9 \mathrm{ml}$ d'eau MilliQ ultra-pure). Les échantillons ont ensuite été entreposés à $4^{\circ} \mathrm{C}$ dans des tubes de polyéthylène de haute densité jusqu'à l'analyse par ICP-MS (model VG2000).

\subsubsection{Biomasse des algues du périphyton}

Six des échantillons de périphyton récoltés ( 3 à l'entrée et 3 à la sortie) ont été conservés congelés pour estimer la biomasse des algues. Pour déterminer si la biomasse était variable entre l'entrée et la sortie, des extractions de chlorophylle a ont été effectuées à l'aide de l'acétone $90 \%$ et de diméthyl sulfoxide (BURNISON 1980). L'absorbance de chaque extrait a été mesurée à l'aide d'un spectrophotomètre UV-visible (SP8-100, PYE UNICAM). La biomasse de chlorophylle a a été calculée selon les équations de JEFFREY et HUMPHREY (1975).

\subsection{Contrôle de qualité}

\subsubsection{Digestions}

Toutes les digestions ont été accompagnées d'un standard et d'un blanc à tous les dix échantillons. Pour la digestion des gastéropodes, le standard de foie de bovin (NIST no 1577B) a été utilisé et le standard de feuilles de pomme a été utilisé (NIST no 1515) pour la digestion du périphyton. Les concentrations de métaux dans les standards étaient dans les limites acceptables (moins de $15 \%$ d'écart de la valeur de référence).

\subsection{2- Eau}

Pour la collecte d'eau, des bouteilles HDPE, des filtres de même que des seringues neuves ont été utilisés sans les baigner préalablement dans $\mathrm{HNO}_{3}$ $15 \%$. HALL (1998) a demontré que ce matériel de collection ne contaminait que de très peu en métaux. Les échantillons d'eau étaient acidifiés à l'aide d'acide nitrique de haute pureté (Seastar Chemicals Inc., Vancouver). A chaque journée d'échantillonnage, trois blancs de terrain ont été faits et chaque fois la valeur de la concentration était sous la limite de detection du ICP-MS; soit $5 \mu \mathrm{g}$

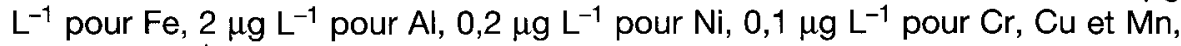
et $0,01 \mu \mathrm{g} \mathrm{L}^{-1}$ pour $\mathrm{Pb}$.

\subsubsection{Substrat artificiel}

Un contrôle en laboratoire a été fait afin d'assurer que les tuiles ne dégageaient aucun métal sous l'effet des sécrétions produites par la flore bactérienne et algale (mucopolysaccharides et glycocalix) (BURKHOLDER, 1996). Nous avons fait incuber une tuile dans un litre d'eau MilliQ durant une période 
de 10 jours à pH 6.0. La solution a ensuite été analysée par ICP-MS (Commission Géologique du Canada) et les concentrations en métaux étaient sous les limites de détection.

\subsection{Rétention de métaux}

L'efficacité de traitement (le pourcentage de la charge de métaux retenue quotidiennement) du marais a été calculée en utilisant la formule suivante :

$$
\begin{gathered}
\text { Efficacité de traitement }(\%)=\left[\left(M_{E}-M_{S}\right) / M_{E}\right] \times 100= \\
{\left[\left(Q c_{E}-Q c_{S}\right) / Q c_{E}\right] \times 100}
\end{gathered}
$$

où $M_{E}$ et $M_{S}$ représentent la masse quotidienne de métaux dissous à l'entrée et à la sortie d'eau du marais $\left(\mu \mathrm{g} \mathrm{j}^{-1}\right), c_{E}$ et $c_{S}$ repésentent les concentrations de métaux dissous à l'entrée et à la sortie d'eau $\left(\mu \mathrm{g} \mathrm{m}^{-3}\right)$ et $Q$ représente le débit $\left(\mathrm{m}^{3} \mathrm{j}^{-1}\right)$ (DORTCH, 1996; WIEDER, 1993). Le débit a été mesuré pour chaque jour d'échantillonnage (GOULET et al., 2001b). À chaque journée d'échantillonnage, les masses pour chaque métal à l'entrée et à la sortie ont été calculées en multipliant la concentration du métal par le débit. En période de débits variables, KADLEC et KNIGHT (1996) suggèrent de grouper les masses à l'entrée et à la sortie correspondant à une période de trois fois la valeur du temps de résidence des eaux (environ un mois dans cette étude). Nous avons donc calculé la moyenne des masses de chaque métal à l'entrée et à la sortie et calculé l'efficacité de traitement.

\subsection{Analyse statistique}

Nous avons fait des tests de $t$ ou de Kruskal-Wallis (si la distribution des données n'était pas normale) pour comparer les concentrations de métaux dissous dans l'eau, de métaux dans le périphyton et dans les tissus de $H$. trivolvis entre l'entrée et la sortie et aussi pour comparer les paramètres chimiques et les concentrations des ions majeurs entre l'entrée et la sortie (logiciel SYSTAT 7.0 pour Windows).

\section{3 - RÉSULTATS}

\subsection{Données météorologiques, hydrologiques et paramètres chimiques}

Au cours des 20 jours de l'expérience, les précipitations, le débit et le temps de résidence de l'eau dans le marais ont été assez variables (tableau 1). Le 6 septembre a été la journée au cours de laquelle il y a eu le plus de précipitations $(\sim 40 \mathrm{~mm})$ de tout le mois de septembre 1999 dans la région d'OttawaCarleton (Environnement Canada). 
Tableau 1 Données hydrologiques et météorologiques pour la période du 3 au 23 septembre 1999.

Table 1 Hydrological parameters and weather conditions for September 3 to 23, 1999.

\begin{tabular}{ccccc}
\hline Date & $\begin{array}{c}\text { Précipitations } \\
(\mathbf{m m})\end{array}$ & $\begin{array}{c}\text { Temps de résidence } \\
\text { hydraulique (jour) }\end{array}$ & $\begin{array}{c}\text { Débit } \\
\left(\mathbf{m}^{3} \mathbf{j o u r} \mathbf{~}^{-1}\right)\end{array}$ & $\begin{array}{c}\mathbf{T} \\
\text { atmosphérique }\left({ }^{\circ} \mathbf{C}\right)\end{array}$ \\
\hline $99-09-03$ & 0 & 31,1 & 1708 & 24,4 \\
$99-09-06$ & 39,8 & 0,83 & 64234 & 23,2 \\
$99-09-08$ & 13,5 & 1,67 & 31768 & 21,1 \\
$99-09-13$ & 3,4 & 9,95 & 5334 & 19,1 \\
$99-09-17$ & Trace & 14,6 & 3729 & 15,3 \\
$99-09-23$ & 0,4 & 10,8 & 4915 & 13 \\
\hline
\end{tabular}

Parmi tous les paramètres chimiques, seule l'alcalinité était significativement différente $(p<0,05)$ entre l'entrée et la sortie. L'alcalinité, pour tous les jours d'échantillonnage, était plus élevée à l'entrée qu'à la sortie. La température de l'eau à la sortie était en moyenne d'environ $3^{\circ} \mathrm{C}$ plus élevée que la température à l'entrée (tableau 2). L'oxygène dissous et le $\mathrm{pH}$ étaient assez variables, même si les données ont été prises au même moment de la journée. Les concentrations moyennes de la majorité des ions étaient similaires ou sensiblement plus elevées à l'entrée et à la sortie (tableau 3).

Tableau 2 Paramètres physico-chimiques (moyenne \pm écart type, $n=6$ ) mesurés durant la période d'échantillonnage. OD: oxygène dissous, SpCond: conductivité spécifique, nd: non disponible. Le symbole * indique une différence significative $(p<0,05)$.

Table 2 Chemical and physical parameters (mean \pm standard error, $n=6$ ) measured during the sampling period. OD: dissolved oxygen, SpCond: conductivity, nd: not available. *indicates a statistically significant difference between inlet and outlet $(p<0,05)$.

\begin{tabular}{lcc}
\hline \multicolumn{1}{c}{ Paramètres } & Entrée (inlet) & Sortie (outlet) \\
\hline $\mathrm{T}\left({ }^{\circ} \mathrm{C}\right)$ & $17,4 \pm 3,2$ & $20,2 \pm 4,6$ \\
$\mathrm{OD}\left(\mathrm{mg} \mathrm{L}^{-1}\right)$ & $7,4 \pm 2,6$ & $7,5 \pm 3,5$ \\
$\mathrm{pH}$ & $7,66 \pm 0,25$ & $7,71 \pm 0,47$ \\
$\mathrm{SpCond}(\mathrm{mS} / \mathrm{cm})$ & $885 \pm 508$ & $727 \pm 148$ \\
Alcalinité $\left(\mu \mathrm{g} \mathrm{L}^{-1}\right)^{*}$ & $5142 \pm 1577$ & $3165 \pm 766$ \\
\hline
\end{tabular}


Tableau 3 Comparaison des concentrations moyennes $(n=6)$ des ions majeurs et du carbone organique dissous (COD) (en mg L-1) entre l'entrée et la sortie du marais durant la période du 3 au 23 septembre 1999.

Table 3 Comparison of the mean $(n=6)$ major ion and dissolved organic carbon concentrations $\left(\mathrm{mg} \mathrm{L}^{-1}\right)$ between inlet and outlet of the wetland for September 3 to 23, 1999.

\begin{tabular}{ccc}
\hline Paramètres & Entrée & Sortie \\
\hline $\mathrm{COD}$ & $6,1 \pm 0,9$ & $6,6 \pm 0,8$ \\
$\mathrm{Ca}^{+}$ & $103,4 \pm 56,2$ & $71,2 \pm 17,9$ \\
$\mathrm{~K}^{+}$ & $4,0 \pm 1,4$ & $3,6 \pm 0,3$ \\
$\mathrm{Mg}^{2+}$ & $19,3 \pm 11,3$ & $16,3 \pm 3,8$ \\
$\mathrm{Na}^{+}$ & $35,7 \pm 19,2$ & $31,7 \pm 7,9$ \\
$\mathrm{Cl}^{-}$ & $73,7 \pm 41,6$ & $65,9 \pm 18,6$ \\
$\mathrm{~F}^{-}$ & $0,20 \pm 0,04$ & $0,19 \pm 0,01$ \\
$\mathrm{~N}^{-}$ & $2,26 \pm 2,04$ & $0,59 \pm 0,52$ \\
$\mathrm{PO}_{4}{ }^{3-}$ & $0,07 \pm 0,05$ & $0,08 \pm 0,07$ \\
$\mathrm{SO}_{4}{ }^{2-}$ & $105,3 \pm 61,1$ & $81,3 \pm 20,7$ \\
\hline
\end{tabular}

\subsection{Balance de masse}

Nous avons calculé l'efficacité de traitement afin de déterminer si le marais était un puit ou une source de métaux (figure 2). En moyenne, le marais retenait une proportion importante de la fraction dissoute de $\mathrm{Cu}, \mathrm{Mn}$, Ni et $\mathrm{Zn}$. Cependant, la rétention de ces metaux était très variable. Les concentrations de Cd étaient trop proche de la limite de détection pour faire un calcul de rétention. Par contre, le marais était peu efficace à retenir la fraction dissoute de $\mathrm{Al}, \mathrm{Fe}$ et $\mathrm{Pb}$ et le marais était une source de $\mathrm{Cr}$ dissous.

Toutes les concentrations de métaux dissous étaient sous les limites établies par les normes provinciales de l'Ontario et fédérales pour la protection de la faune aquatique d'eau douce (fig. 3, 4) (MEEO, 1994 ; CCME, 1999).

\subsection{Concentrations de métaux dans le périphyton et les gastéropodes}

De façon générale, les concentrations des métaux essentiels (figure 3) et non-essentiels (figure 4) étaient plus élevées dans le périphyton que dans les tissus des gastéropodes. Seules les concentrations de $\mathrm{Cd}$ et de $\mathrm{Zn}$ étaient plus élevées dans les tissus des gastéropodes.

II y avait trois tendances principales pour les concentrations de métaux dans le périphyton. Premièrement, les concentrations moyennes de $\mathrm{Zn}$ et de certains métaux non-essentiels $(\mathrm{Cd}, \mathrm{Ni})$ étaient significativement $(p<0,05)$ plus élevées à l'entrée qu'à la sortie. Deuxièmement, les concentrations moyennes de $\mathrm{Fe}$ et $\mathrm{Mn}$ étaient significativement $(p<0,05)$ plus élevées à la sortie qu'à 


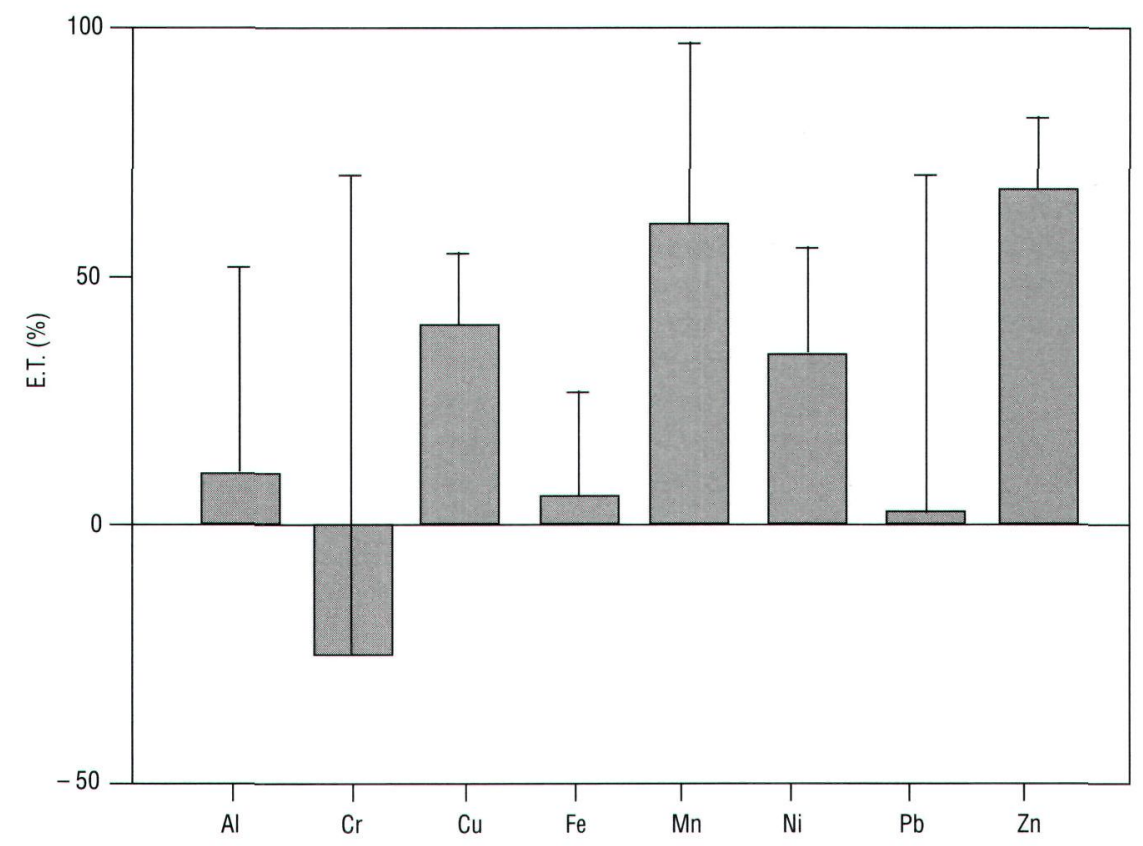

Figure 2 Efficacité de traitement (E.T.) moyenne (Les barres d'erreur correspondent aux écarts-type) de 8 métaux dissous pour la période du 3 au 23 septembre 1999.

Mean Treatment Efficiency (E.T.) (Error bass correspond to standard erros for 8 dissolved metals for september 8 to 23, 1999.

l'entrée. Finalement, les concentrations en $\mathrm{Cu}, \mathrm{Cr}$ et $\mathrm{Al}$ du périphyton à l'entrée et à la sortie étaient similaires.

Pour les gastéropodes, le diamètre de leurs coquilles variait de 13 à $19 \mathrm{~mm}$ à l'entrée et de 14 à $21 \mathrm{~mm}$ à la sortie. II n'y avait pas de différence significative dans la taille des gastéropodes entre l'entrée et la sortie (test de t, $p=0,472$; diamètre moyen de la coquille: 16,8 $\mathrm{mm}$ (entrée) et 17,3 $\mathrm{mm}$ (sortie)).

II y avait trois principales tendances pour les concentrations de métaux dans les gastéropodes (figures 3-4). Premièrement, les concentrations moyennes de $\mathrm{Cd}$, $\mathrm{Ni}$ et $\mathrm{Pb}$ étaient significativement plus élevées à l'entrée qu'à la sortie du marais. Deuxièmement, les concentrations en $\mathrm{Al}$ et $\mathrm{Cr}$ à l'entrée et à la sortie du marias étaient similaires. Finalement, les concentrations moyennes de $\mathrm{Cu}, \mathrm{Fe}$, et $\mathrm{Mn}$ étaient plus élevées à la sortie qu'à l'entrée du marais. 

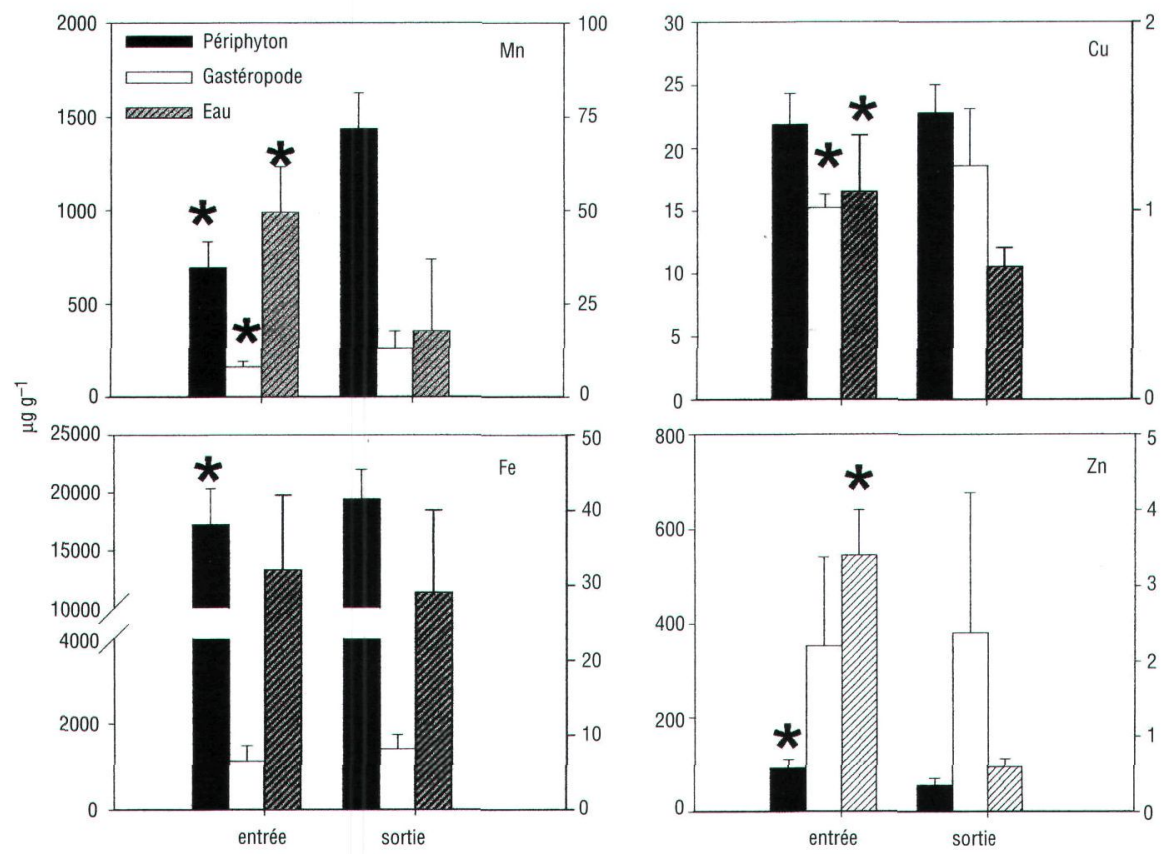

Figure 3 Concentrations moyennes de 4 métaux essentiels dissous dans le périphyton, les gastéropodes (sur l'échelle de gauche de chaque graphique) et l'eau (sur l'échelle de droite de chaque graphique), à l'entrée et à la sortie d'eau du marais pour la période du 3 au 23 septembre 1999 (les barres d'erreur correspondent aux écartstypes). Normes canadiennes de la qualité des eaux pour la protection de la vie aquatique: $\mathrm{Cu}=2 \mu \mathrm{g} \mathrm{L}^{-1}, \mathrm{Fe}=300 \mu \mathrm{g} \mathrm{L}^{-1}, \mathrm{Zn}=30 \mu \mathrm{g} \mathrm{L}^{-1}$ (CCME 1999). * correspond à une différence significative $P<0,05$ entre les concentrations en métaux à l'entrée et à la sortie.

Mean dissolved concentrations of 4 essential metals in periphyton, gastropods ( scale on left of each graph) and water ( scale on right of each graph) at the inlet and outlet of the wetland for September 3 to 23, 1999 (Error bars correspond to standard errors). Canadian water quality guidelines for the protection of aquatic life: $\mathrm{Cu}=2 \mu \mathrm{g} \mathrm{L^{-1 }}, \mathrm{Fe}=$ $300 \mu g L^{-1}, Z n=30 \mu g L^{-1}$ (CCME 1999). * corresponds to significant differences $P<0,05$ in metal concentrations between the inlet and outlet. 

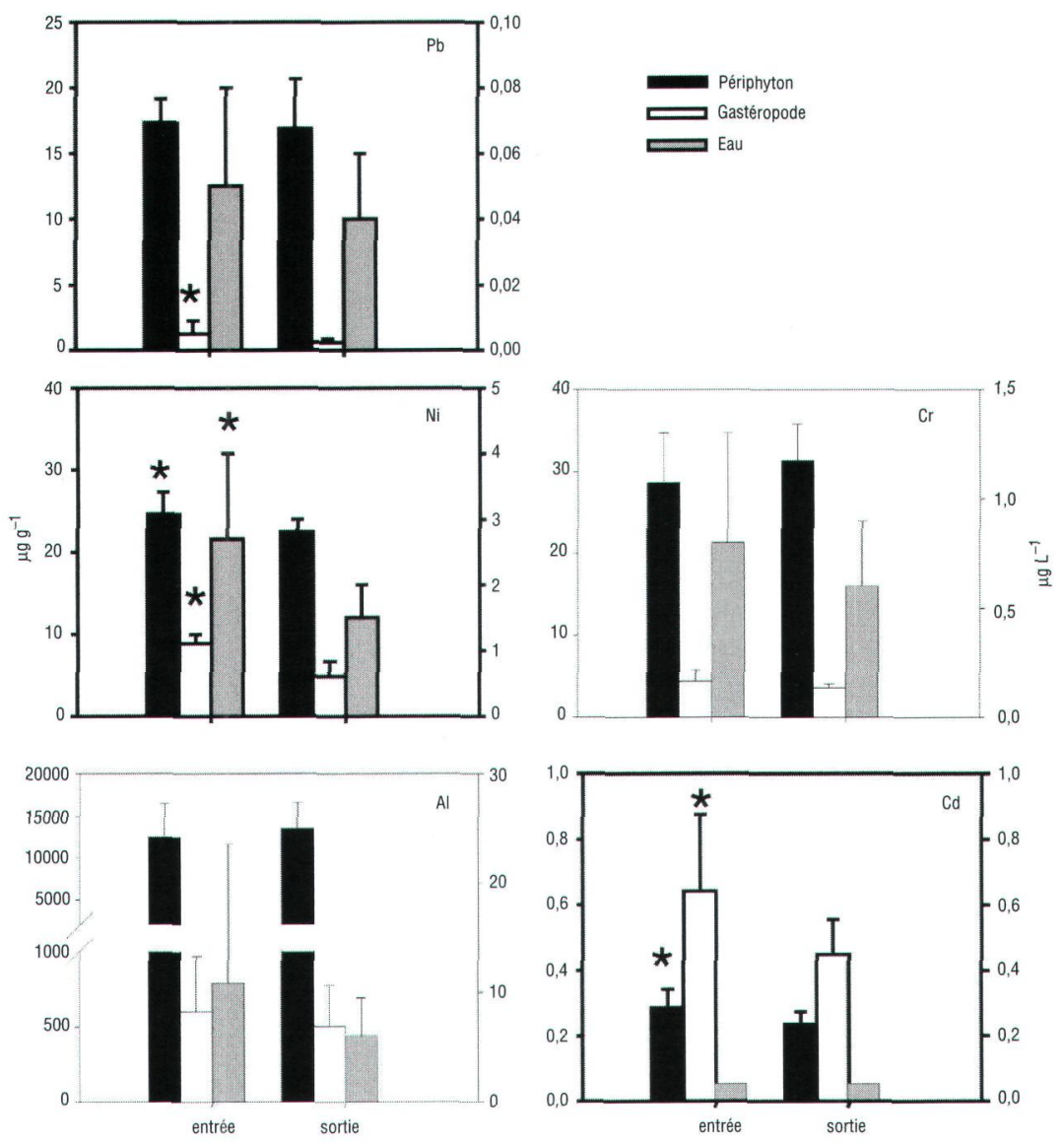

Figure 4 Concentrations moyennes de 5 métaux non-essentiels dans le périphyton, les gastéropodes et l'eau, à l'entrée et à la sortie d'eau du marais pour la période du 3 au 23 septembre 1999 (les barres d'erreur correspondent aux écarts-types). Normes canadienne de la qualité des eaux pour la protection de la vie aquatique: $\mathrm{Al}=100 \mathrm{mg} \mathrm{L}^{-1}$ $\mathrm{Cd}=0,017 \mu \mathrm{g} \mathrm{L}^{-1}, \mathrm{Cr}(\mathrm{VI})=1.0 \mu \mathrm{g} \mathrm{L}-1, \mathrm{Ni}=65 \mu \mathrm{g} \mathrm{L}-1, \mathrm{~Pb}=2 \mu \mathrm{g} \mathrm{L}^{-1}$ (CCME 1999). * correspond à une différence significative $\mathrm{P}<0,05$ entre les concentrations en métaux à l'entrée et à la sortie.

Mean concentrations of 5 non-essential metals in periphyton, gastropods and water at the inflow and outflow of the wetland for September 3 to 23, 1999 (Error bars correspond to standard errors). Canadian water quality guidelines for the protection of aquatic life: Al $=100 \mu g L^{-1}, C d=0,017 \mu g L^{-1}, C r(V I)=1.0 \mu g L^{-1}, N i=65 \mu g L^{-1}$, $P b=2 \mu g L^{-1}$ (CCME 1999). * corresponds to significant $P<0,05$ differences in metal concentrations between the inlet and outlet. 


\subsection{Analyse de biomasse}

La biomasse moyenne de chlorophylle a du périphyton était significativement plus élevée à la sortie $\left(73,6 \mathrm{mg} / \mathrm{m}^{2}\right)$ qu'à l'entrée $\left(43,3 \mathrm{mg} / \mathrm{m}^{2}\right)$ du marais (test de t, $p=0,043$ ).

\section{4 - DISCUSSION}

\subsection{Concentrations en métaux de $\boldsymbol{H}$. trivolvis}

$H$. trivolvis pourrait être utilisé comme biomoniteur des métaux non essentiels ( $\mathrm{Al}, \mathrm{Cd}, \mathrm{Cr}, \mathrm{Ni}, \mathrm{Pb}$ ) dans le marais, puisqu'on observe des tendances similaires entre les niveaux de ces métaux dissous dans l'eau et dans les tissus des individus. Selon METCALFE-SMITH et al. (1996), un bon biomoniteur devrait avoir des concentrations internes corrélées avec les niveaux d'exposition. Par contre, $H$. trivolvis semble être un mauvais biomoniteur des métaux essentiels $(\mathrm{Cu}, \mathrm{Fe}$, $\mathrm{Mn}, \mathrm{Zn}$ ) dans le marais, puisqu'on n'observe pas des patrons similaires entre les niveaux de ces métaux dans les gastéropodes et les niveaux dans l'eau.

Ces deux tendances opposées pourraient être reliées aux processus métaboliques. Les mollusques possédent la capacité de réguler leurs concentrations internes de métaux essentiels (LANGSTON et al., 1998). AMIARD et al. (1987) ont aussi observé que les gastéropodes et les bivalves avaient la capacité de maintenir leurs concentrations internes de métaux relativement constantes, quand les concentrations dans le milieu changeaient. HARE et TESSIER (1998) ont observé que la larve de l'insecte aquatique Chaoborus punctipennis était capable de maintenir ses concentrations internes de $\mathrm{Cu}$ et de $\mathrm{Zn}$ relativement constantes par rapport aux concentrations dans l'environnement. Cette capacité de régulation de $\mathrm{Cu}$ et $\mathrm{Zn}$ a d'ailleurs été observé chez plusieurs invertébrés aquatiques (AMIARD et al., 1987).

II est aussi possible que les concentrations de métaux essentiels (Fe, Mn, $\mathrm{Cu}$ et $\mathrm{Zn}$ ) dissous dans l'eau du marais ne soient pas une bonne indication de la forme biodisponible aux gastéropodes et le périphyton. GOULET et al. (2001a) ont observé que le contenu en Fe et $\mathrm{Mn}$ de $H$. trivolis était relié à la forme particulaire de $\mathrm{Fe}$ et $\mathrm{Mn}$, plutôt qu'à la forme dissoute. Les gastéropodes contenaient plus de $\mathrm{Fe}$ et de $\mathrm{Mn}$ à la sortie qu'à l'entrée car le marais Monahan était une source de $\mathrm{Fe}$ et de Mn particulaire.

Par ailleurs, les gastéropodes ont été exposés à l'eau durant une période beaucoup plus longue que le périphyton. Les gastéropodes pulmonés d'eau douce que l'on retrouve dans les régions tempérées ont un cycle de vie annuel (CALOW, 1983). Par conséquent, les concentrations que l'on observe dans les tissus de $H$. trivolvis ne reflètent pas seulement les concentrations de métaux mesurées durant la période d'échantillonnage. GOULET et al. (2001a) ont observé que les teneurs en métaux d' $H$. trivolis varient de façon saisonnière. Cette variation peut provenir d'un changement dans la teneur en métaux de sa 
nourriture puisque les gastéropodes pulmonés y puisent principalement leurs métaux (FLESSAS et al., 2000). HARE et CAMPBELL (1992) ont aussi observé que les changements saisonniers du contenu en métaux de Chaborus punctipennis étaient reliés à ceux de sa nourriture.

\subsection{Concentrations en métaux du périphyton}

Les concentrations étaient beaucoup plus élevées dans le périphyton que dans les tissus de $H$. trivolvis, excepté pour le $\mathrm{Cd}$ et le $\mathrm{Zn}$. Pour ces métaux, nos résultats sont comparables à ceux obtenus par MAZLANI et al. (1994) dans leur étude sur le gastéropode prosobranche d'eau douce Melanopsis praemorsa. Ils ont observé des concentrations inférieures de $\mathrm{Cd}$ et de $\mathrm{Zn}$ dans le périphyton comparativement aux tissus de $M$. praemorsa. Le Cd aurait la capacité de traverser les membranes via des canaux de calcium et on a aussi observé qu'il se liait aux métallothionines chez le gastéropode Littorina littorea (LANGSTON et al., 1998).

Les études en laboratoire et sur le terrain indiquent qu'il est possible de généraliser les processus d'assimilation des métaux des algues planctoniques et des algues associées à un substrat (STOKES et al., 1983). Les algues peuvent accumuler des concentrations d'ions inorganiques des milliers de fois plus élevées que les concentrations dans leur environnement par divers mécanismes biologiques, chimiques et physiques impliquant l'adsorption, la précipitation et des processus métaboliques (GENTER, 1996). Les polysaccharides chargés négativement de la cellule algale seraient d'ailleurs en partie responsables de l'accumulation de certains métaux par une simple réaction d'échange d'ions. La forme disponible des métaux comme le $\mathrm{Cu}$, le $\mathrm{Zn}$, le $\mathrm{Ni}$ et le $\mathrm{Cd}$ est normalement l'ion libre ou l'ion hydraté (SANDERS et RIEDEL, 1998; STOKES et al., 1983). Le périphyton peut jouer le rôle de surface «adhérante » pour plusieurs contaminants; il a tendance à concentrer les contaminants soit par assimilation ou par adsorption (BOSTON, 1993). L'ingestion de périphyton contaminé peut donc être une source importante de contaminants pour certains brouteurs (BOSTON, 1993; GENTER, 1996).

Le périphyton que l'on retrouve sur les substrats rocheux obtient ses nutriments directement de l'eau à sa surface ou de la régénération microbienne qui s'effectue dans la matrice de périphyton (BURKHOLDER, 1996). C'est pourquoi des tendances similaires entre les concentrations de métaux à l'entrée et à la sortie du marais dans le périphyton et dans l'eau ont été observées (figure 3). Par contre, les concentrations de certains métaux essentiels ( $\mathrm{Cu}, \mathrm{Fe}, \mathrm{Mn}$ ) à l'entrée et à la sortie du marais dans le périphyton n'étaient pas directement liées aux concentrations dissoutes. Les concentrations de $\mathrm{Fe}, \mathrm{Mn}$, et $\mathrm{Cu}$ dans le périphyton étaient plus elevées à la sortie du marais. Ce phénomene pourrait s'expliquer en partie par une biomasse de périphyton plus élevée à la sortie du marais où les concentrations en phosphates étaient plus élevées. Plusieurs études indiquent que le taux de croissance peut influencer l'assimilation des métaux pas les algues (; ENGDAHI et al., 1998; GENTER, 1996; PETERSON et NYHOLM 1993). En fait, la quantité de métaux accumulés par les algues augmente avec la biomasse, mais à des biomasses très élevées, le pourcentage de métal accumulé peut diminuer ou demeurer stable (GENTER, 1996). Cependant, pour les métaux non essentiels, ce phénomène n'a pu être observé peutêtre parce que l'assimilation de métaux non essentiels ne dépend pas autant 
des processus métaboliques. GENTER (1996) affirme aussi que les métaux essentiels peuvent être accumulés à des niveaux différents que les métaux non essentiels par le périphyton.

\section{5 - CONCLUSION}

L'utilisation du périphyton sur substrat artificiel, tout particulièrement, apparaît comme un moyen pratique et peu coûteux d'évaluer la qualité des eaux. De plus, la durée de croissance du périphyton étant connue, il peut donc reflèter les niveaux de contamination pour différentes périodes d'exposition. L'échantillonnage du périphyton est aussi une méthode plus conservatrice de protéger les organismes vivants, étant donné que les teneurs en métaux sont généralement plus élevées dans le périphyton que dans les gastéropodes.

Notre étude montre aussi l'importance du dosage de métaux dans des organismes aquatiques afin d'évaluer plus exactement l'efficacité des marais artificiels à retenir les métaux dissous. L'utilisation exclusive de données chimiques de concentrations des métaux dissous ne semble pas être une méthode appropriée pour évaluer la qualité des eaux traitées par les marais artificiels.

\section{REMERCIEMENTS}

Les auteurs tiennent à remercier Gwendy Hall et Judy Vaive du Centre Géologique du Canada pour les analyses de métaux par ICP-MS. Les analyses des anions ont été faites par John Loop au Département de Géologie de I'Université d'Ottawa. Cette étude a été réalisée grâce à une subvention de recherche interfacultaire avec R. Droste (Faculté de génie) et D. Fortin (Faculté des sciences).

\section{RÉFÉRENCES BIBLIOGRAPHIQUES}

AMIARD J.C., AMIARD-TRIQUET C., BERTHET B., METAYER C., 1987. Comparative study of the patterns of bioaccumulation of essential $(\mathrm{Cu}, \mathrm{Zn})$ and non essential ( $\mathrm{Cd}, \mathrm{Pb}$ ) trace metals in various estuarine and coastal organisms. J. Exp. Mar. Biol. Ecol., 106, 73-89.
BOSTON H.L., 1993. Grazers, periphyton and toxicant movement in streams. Environ. Toxicol. Chem., 12, 955-957.

BOYDEN C.R., 1974. Trace element content and body size in molluscs. Nature, 251, 311-314. 
BREEN P.F., MAG V., SEYMOUR B.S., 1994. The combination of a flood-retarding basin and a wetland to manage the impact of urban runoff. Wat. Sci. Technol., 29, 103-109.

BROWN K. M., 1991. Mollusca: Gastropoda. In "Ecology and classification of North American freshwater invertebrates ", THORP J.H. et COVICH A.P. [éds], Academic Press Inc., San Diego, CA, pp. 285-314.

BURKHOLDER J.M., 1996. Interaction of benthic algae with their substrata. In «Algal ecology: freshwater benthic ecosystems ", STEVENSON R.J., BOTHWELL M.L. et LOWE R.L. [éds.], Academic Press, San Diego, CA, pp. 253-297.

BURNISON K., 1980. Modified dimethylsulfoxide (DMSO) extraction for chlorophyll analysis of phytoplankton. Can. J. Fish Aquat. Sci., 37, 729-733.

CALOW, P., 1983. Life-cycle patterns and evolution, In «The Mollusca", RUSSELLHUNTER W.D. et WILBUR K.M. [éds], Academic Press, Orlando, Florida. pp. 649-678.

CCME. 1999. Recommandations canadiennes pour la qualité de l'environnement. Le Conseil Canadien des Ministres de l'Environnement, Winnipeg, Canada.

CITY OF KANATA, 1997. Monahan drain constructed wetlands: 1997 monitoring report. Totten Sims Hubicki \& Associates, City of Kanata, ON. 14 p.

CITY OF KANATA, 1998. Monahan drain constructed wetlands: 1998 monitoring report. Totten Sims Hubicki \& Associates, City of Kanata, ON. 15 p.

COUGHTREY P.J., MARTIN M.H., 1977. The uptake of lead, zinc, cadmium, and copper by pulmonate mollusc, Helix asperga Muller, and its relevance to the monitoring of heavy metal contamination of the environment. Oecologia, 27, 65-74.

DORTCH M.S., 1996. Removal of solids, nitrogen, and phosphorus in the Cache River wetland. Wetlands, 16, 358-365.

ENGDAHI S., MAMBOYA F., MTOLERA M., SEMESI A., BJORK M., 1998. The brown macroalgae Padina boergesenii as an indicator of heavy metal contamination in the Zanzibar channel. Ambio, 27, 694700 .
FLESSAS, C., COUILLARD, Y., PINELALLOUL, B., ST-CYR, L., CAMPBELL P.G.C., 2000. Metal concentrations in two freshwater gastropods (Mollusca) in the St-Lawrence River and relationships with environmental contamination. Can. J. Fish. Aquat. Sci. , 57, 1-12.

GENTER B.G., 1996. Ecotoxicology of inorganic chemical stress to algae. In « Algal ecology: freshwater benthic ecosystems ", STEVENSON, R.J., BOTHWELL, M.L. et LOWE, R.L. [Ed.], Academic Press, San Diego, CA, pp.403-468.

GOULET R.R., PICK F.R., 2001. Diel changes in iron concentrations in the surface waters in surface-flow constructed wetlands. Water Sci. Technol., 44, 421-426.

GOULET R.R., LECLAIR E.N., PICK F.R., $2001 a$. The evaluation of metal retention by a constructed wetland using the plumonate gastropod Helisoma trivolis (Say). Arch. Environ. Contam. Toxicol., 40, 303-310.

GOULET R.R., PICK F.R., DROSTE R. L., $2001 \mathrm{~b}$. Test of the first order removal model for metal retention in a young constructed wetland. Ecol. Engineering $17,357-371$.

HALL G.E.M., 1998. Cost-effective protocols for the collection, filtration and preservation of surface water for detection of metals and metalloids at ppb $\left(\mu \mathrm{g} \mathrm{L}^{-1}\right)$ and ppt (ng $L^{-1}$ ) levels. Phase I: Evaluation of bottle type, bottle cleaning, filter and preservation technique. Aquatic Effects Technology Evaluation Program (Task Force on Water Quality Issues), CANMET, Ottawa, Ont., AETE Projects 3.1.3. Natural Resources Canada, $193 \mathrm{p}$.

HARE L., TESSIER A., 1998. The aquatic insect Chaoborus as a biomonitor of trace metals in lakes. Limnol. Oceanogr., 43, 1850-1859.

HARE L., CAMPBELL P.G.C., 1992. Temporal variations of trace metals in aquatic insects. Freshwater Biol., 27, 13-27.

HAWKINS W.B., RODGERS J.H.JR., DUNN A.W., DORN P.B., CANO M.L., 1997. Design and construction of wetlands for aqueous transfers and transformations of selected metals. Ecotoxicol. Environ. Saf., 36, 238-248.

HELFIELD J.M., DIAMOND M.L., 1997. Use of constructed wetlands for urban 
stream restoration: a critical analysis. Environ. Manag., 21, 329-341.

JEFFREY S.W., HUMPHREY G.F., 1975. New spectrophotometric equations for determining chlorophylls $\mathrm{a}, \mathrm{b}, \mathrm{c} 1$ and $\mathrm{c} 2$ in higher plants, algae and natural phytoplankton. Biochem. Physiol. Planzen., 167, 191-194.

KADLEC R.H., KNIGHT R.L., 1996. «Treatment Wetlands»; Lewis Publisher, Boca Raton, FI, 893 p.

LANGSTON W.J., BEBIANNO M.J., BURT G.R., 1998. Metal handling strategies in molluscs. In " Metal metabolism in aquatic environments ", Langston W.J. et Bebianno M.J. [éds.], Chapman \& Hall, New York, NY, pp. 292-319.

MAZLANI S., MAAROUF A., RADA A., EL MERAY M., PIHAN J.C., 1994. Étude de la contamination par les métaux lourds du champ d'épandage des eaux usées de la ville de Marrakech (Maroc). Rev. Sci. Eau, 7, 55-68.

METCALFE-SMITH J.L., GREEN R.H., GRAPENTINE L.C., 1996. Influence of biological factors on concentrations of metals in the tissues of freshwater mussel (Elliptio complanata and Lampsillis radiata radiata) from the St. Lawrence River. Can. J. Fish. Aquat. Sci., 53, 205-219.

MITSCH W.J., GOSSELINK J.G., 2000. “Wetlands", John Wiley \& Sons, Inc., New York, 920 p.

MEEO. Ministère de l'Environnement et de l'Énergie de l'Ontario, 1994. Water management policies guidelines, provincial water quality objectives. (http://www.ene.gov.on.ca/)

PETERSON H.G., NYHOLM N., 1993. Algal bioassays for metal toxicity identification. Water Pollut. Res. J. Can., 28, 129-153.

REDDY K.R., KADLEC R.H., FLAIG E., GALE P.M., 1999. Phosphorus retention in streams and wetlands: a review. Crit. Rev. Environ. Sci. Technol., 29, 83-146.

SANDERS J.G., RIEDEL G.F., 1998. Metal accumulation and impacts in phytoplankton. In « Metal metabolism in aquatic environments ", LANGSTON W.J. et BEBIANNO M.J. [éds.], Chapman \& Hall, New York, NY, pp. 59-76.

SCHOLES L., SHUTES R.B.E,, REVITT D.M., FORSHAW M., PURCHASE D., 1998. The treatment of metals in urban runoff by constructed wetlands. Sci. Total Environ. 214, 211-219.

SMITH D.A., 1989. Tests of feeding selectivity in Helisoma trivolvis Gastropoda Pulmonata. Trans. Am. Mircroscopical Soc., 108, 394-402.

STOKES P.M., DREIER S.I., FARKAS M.O., MCLEAN R.A.N., 1983. Mercury accumulation by filamentous algae: a promising biological monitoring system for methyl mercury in acid-stressed lakes. Envron. Pollut., 5, 255-271.

VYMAZAL J., 1995. "Algae and element cycling in wetlands ", $4^{\mathrm{e}}$ édition; LEWIS PUBLISHERS, Boca Raton, FI, 689 p.

WETZEL R., LIKENS G., 1991. «Limnological analyses ", $2^{\oplus}$ édition; SPRINGER-VERLAG, New York, NY, 391 p.

WIEDER R.K., 1993. Ion input/output budgets for five wetlands constructed for acid coal mine drainage treatment. Water Air Soil Pollut. 71, 231-270.

WIEDER R.K., 1994. Diel changes in iron(III)/iron(II) in effluent from constructed acid mine drainage wetlands. J. Environ. Qual., 23, 730-738.

WOOD R.B., MCATAMNEY C.F., 1996. Constructed wetlands for wastewater treatment: the use of laterite in the bed medium in phosphorus and heavy metal removal. Hydrobiologia, 340, 323-331. 